\title{
On Meyniel's conjecture of the cop number
}

\author{
Linyuan $\mathrm{Lu}^{*} \quad$ Xing Peng ${ }^{\dagger}$
}

March 22, 2009

\begin{abstract}
Meyniel conjectured the cop number $c(G)$ of any connected graph $G$ on $n$ vertices is at most $C \sqrt{n}$ for some constant $C$. In this paper, we prove Meyniel's conjecture in special cases that $G$ has diameter at most 2 or $G$ is a bipartite graph with diameter at most 3 . For general connected graphs, we prove $c(G)=O\left(\frac{n}{2^{(1-o(1)) \sqrt{\log _{2} n}}}\right)$, improving the best previously known upper-bound $O\left(\frac{n}{\ln n}\right)$ due to Chiniforooshan.
\end{abstract}

\section{Introduction}

The $\operatorname{cop}(\mathrm{s})$-robber game is played by two players (denoted by $\mathcal{C}$ and $\mathcal{R}$ ) on the board of a simple connected graph $G$ on $n$ vertices. Initially, $\mathcal{C}$ first puts $c$ pebbles (called cops) on vertices of $G$, then $\mathcal{R}$ put one pebble (called the robber) on some vertex. Two players play moves alternatively. At $\mathcal{C}$ 's turn, he (or she) can have each cop stay at its current position or move to its neighbor vertex. Multiple cops are allowed to be placed on the same vertex. At $\mathcal{R}$ 's turn, he (or she) can do nothing or move the robber to a neighbor vertex of its current position. Whenever a cop meets the robber at the same vertex, $\mathcal{C}$ wins. Both players have the complete information of the game board (the graph $G$ and the current positions of cops and the robber). If $\mathcal{C}$ has a winning strategy using $c$ cops, then we say $G$ is $c$-searchable. The cop number $c(G)$ is the minimum integer $c$ so that $G$ is $c$-searchable.

The cop-robber game was first studied by Nowakowski and Winkler [9], and by Quilliot [10]. In both case they classified all graphs on which one cop can win. Aigner and Fromme [1] introduced the concept of the cop number $c(G)$ and proved $c(G) \leq 3$ for any connected planar graph $G$. Since then, many good upper bound on $c(G)$ for special classes of graphs were discovered (see Alpach's survey paper [2]).

Little is known for the cop number of the general connected graph. Meyniel made the following conjecture in personal communication to Frankl [8].

\footnotetext{
*University of South Carolina, Columbia, SC 29208, (lu@math.sc.edu). This author was supported in part by NSF grant DMS 0701111.

$\dagger$ University of South Carolina, Columbia, SC 29208, (pengx@mailbox.sc.edu). This author was supported in part by NSF grant DMS 0701111.
} 
Meyniel's conjecture: There is a constant $C$ so that the cop number of any connected graph $G$ on $n$ vertices satisfying

$$
c(G) \leq C \sqrt{n} .
$$

In this paper, we prove two special cases of Meyniel's conjecture.

Theorem 1 Let $G$ be a connected graph on $n$ vertices. If the diameter of $G$ is at most 2 or $G$ is a bipartite graph with diameter at most 3 , then the cop number $c(G)$ satisfies

$$
c(G) \leq 2 \sqrt{n}-1 .
$$

This result is tight up to a constant multiplicative factor. The constant 2 here can not replaced by $\frac{\sqrt{2}}{2}$ as shown by this following example.

For $q$ a power of some prime, it is well-known that the projective plane of order $q$ exists. View the projective plane as a bipartite graph $G$ with points and lines as vertices and incidence relations as edges. Then $G$ is a $q+1$-regular bipartite graph on $n=2\left(q^{2}+q+1\right)$ vertices (lines and points) with girth 6 . It is also clear that the diameter of $G$ is 3. Aigner and Fromm [1] proved that if $G$ has girth at least 5 then

$$
c(G) \geq \delta,
$$

where $\delta$ is the minimum degree of $G$. Thus, the cop number of $G$ is at least $c(G) \geq q+1>\frac{\sqrt{2}}{2} \sqrt{n}$.

The first sub-linear upper bound for general connected graph $G$ is due to P. Frankl [8], who proved

$$
c(G) \leq(1+o(1)) \frac{n \ln \ln n}{\ln n} .
$$

This bound was recently improved by Chiniforooshan [7]:

$$
c(G) \leq O\left(\frac{n}{\ln n}\right) .
$$

For the random graphs $G(n, p)$, Bollobás, Kun and Leader [6] shows

$$
c(G(n, p))=O(\sqrt{n} \log n)
$$

if $p \geq 2.1 \frac{\log n}{n}$. Luczak-Prałat [12] further determined $c(G(n, p))$ up to a poly$\log -\mathrm{n}$ multiplicative factor.

In this paper, we proved

Theorem 2 For any connected graph $G$, we have

$$
c(G) \leq n 2^{-\left(1-o_{n}(1)\right) \sqrt{\log _{2} n}} .
$$

The paper is organized as follows. In section 2, necessary notations and lemmas are introduced. In section 3, we examine the cop number of graphs with diameter 2 or bipartite graphs with diameter 3 . Theorem 2 on general graphs will be proved in section 4 . 


\section{Notations and Lemmas}

Let $G=(V, E)$ be a finite, simple and connected graph. For any two vertices $u, v$, let $d^{G}(u, v)$ be the graph distance between $u$ and $v$. For any $i \geq 1$ and any set $S$, we define the $i$-th open and closed neighbors of $S$ as

$$
\begin{aligned}
\Gamma_{i}^{G}(S) & =\left\{u \mid \exists v \in S, d^{G}(u, v)=i\right\} \\
N_{i}^{G}(S) & =\left\{u \mid \exists v \in S, d^{G}(u, v) \leq i\right\} .
\end{aligned}
$$

When the graph $G$ is clear in the context, we omit the superscript of $G$ and simply write $d(u, v), \Gamma_{i}(S)$, and $N_{i}(S)$. In the case $S=v$, we simply write $\Gamma_{i}(v)$ for $\Gamma_{i}(\{v\})$ and $N_{i}(v)$ for $N_{i}(\{v\})$. We also omit the subscript $i$ when $i=1$.

We say a cop can control a set $S$ means, after a finite number of moves, if the robber moves onto $S$ at any time $t$, he will be caught at time $t+1$ by this cop. For example, a cop can control $N(v)$ - the set consisting of the neighbors of $v$ and $v$ itself. Ailgner-Fromme [1] proved the following useful lemma.

Lemma 1 (Aigner-Fromme [1]) If $P$ is a shortest path between $u$ and $v$ in $G$, then one cop can control $P$.

The following lemma is a variation of Hall's matching theorem.

Lemma 2 Let $H$ be a bipartite graph with the vertex partition $V(H)=L \cup R$. Then there exists a set $S \subset L$ and a matching $M$ satisfying

1. $S=\emptyset$ or $|\Gamma(S)|<|S|$.

2. $M$ covers all vertices in $L \backslash S$.

Proof: If $|\Gamma(T)| \geq|T|$ for any $T \subset L$. Then we set $S=\emptyset$. In this case, by the Hall's Theorem, we have a matching $M$ covers $L$. Otherwise, there is some $T$ such that $|\Gamma(T)|<|T|$. Then we take $S$ be a maximal one with respect to inclusion. (In general $S$ is not unique.) We have $|\Gamma(S)|<|S|$.

Claim: $|\Gamma(W)| \geq|W|$ for any $W \subset L \backslash S$.

If it is not true. Then there is some $W \subset L \backslash S$ such that $|\Gamma(W)|<|W|$. So we have

$$
|\Gamma(S \cup W)|=|\Gamma(S) \cup \Gamma(W)| \leq|\Gamma(S)|+|\Gamma(W)|<|S|+|W|=|S \cup W| .
$$

The last equality follows from $W \subset L \backslash S$. Hence $S \subset S \cup W$, which contradicts the maximality of $S$. By the Hall's theorem, there is a matching $M$ covers $L \backslash S$.

Let $\Delta$ be the maximum degree and $D$ be the diameter of $G$. The Moore bound says

$$
n \leq 1+\sum_{i=1}^{D} \Delta(\Delta-1)^{i} .
$$


So the diameter $D$ and the maximum degree $\Delta$ can not be both less than $(1+o(1)) \frac{\ln \ln n}{\ln n}$. Put one cop to control a set $S$ as large as possible (so $|S|>$ $\left.(1+o(1)) \frac{\ln \ln n}{\ln n}\right)$. Let $G_{\bar{S}}$ be the induced graph of $G$ on the complement set $\bar{S}$. $G_{\bar{S}}$ may be disconnected. The robber is confined into the the connected component (denoted by $G^{\prime}$ ) of $G_{\bar{S}}$. Now send the rest of cops to $G^{\prime}$. It is clear that

$$
c(G) \leq 1+c\left(G^{\prime}\right) .
$$

Frankl's result (1) is a consequence of this greedy algorithm.

\section{Graphs with small diameter}

In this section, we assume $G$ has diameter at most 2 or $G$ is a bipartite graph with diameter at most 3 . We will prove Meyniel's conjecture holds in this case.

First, we will prove the following lemma. A graph $H$ is called $k$-degenerated if every subgraph of $H$ has a vertex with degree at most $k$.

Lemma 3 Suppose $H$ is a $k$-degenerated subgraph of a connected graph $G$. A variation of the cop-robber game is played on $G$ so that cops are allowed to move along the edges of $G$ while the robber is only allowed to move along the edges of $H$. Initially the robber is on some vertex of $H$. Suppose that $G$ has diameter at most 2 or $G$ is a bipartite graph with diameter 3 . Then $k$ cops can capture the robber after finite steps.

Remark: This lemma is tight ( $k$ can not be replaced by $k-1)$. Let $G$ be the bipartite graph for finite projective plane of order $q$. Then $G$ is a $q+1$-regular graph with diameter 3 . We have shown that

$$
c(G) \geq q+1 .
$$

Apply this lemma to the subgraph $H=G$. Clearly $H$ is $q+1$-degenerated. We note $q+1$ cops can capture the robber after finite steps. So we have

$$
c(G)=q+1 .
$$

Proof of Lemma 3: We will prove by induction on the order of $H$.

If $H$ has at most $k$ vertices, the statement holds trivially. Just send $k$ cops to cover all vertices of $H$.

Suppose $k$ cops can capture the robber after finite steps for any $k$-degenerate subgraph $H$ of order up to $m$.

Now consider a $k$-degenerated subgraph $H$ of order $m+1$. By the definition of $k$-degenerated graph, $H$ has a vertex $v$ of degree at most $k$. It is clear that $H \backslash\{v\}$ is also $k$-degenerated. If the robber never moves to the vertex $v$, he is restricted to the subgraph $H \backslash\{v\}$. By the inductive hypothesis, it is captured by $k$ cops after finite steps. After a while, the robber is forced into the position of $v$. At this moment, say the cops are at the vertices $u_{1}, u_{2}, \ldots, u_{k}$. Say the neighbors of $v$ in $H$ are $v_{1}, v_{2}, \ldots, v_{l}(l \leq k)$. If for some $i, j, u_{i}=v_{j}$, then the 
next step move the cop at $u_{i}$ to $v$ and catch the robber. Now we assume $u_{i} \neq v_{j}$ for any $1 \leq i \leq k$ and $1 \leq j \leq l$.

When the diameter of $G$ is at most 2 , for each $i$, there is a path $P_{i}$ of length at most 2 connecting $u_{i}$ to $v_{i}$. (Here, note that the cops are allowed to move along the edges of $G$.) For $1 \leq i \leq l$, move $i$-th cop at $u_{i}$ along the path $P_{i}$ one step toward $v_{i}$. Each $v_{i}$ is now under the control of $i$-th cops. The robber has no way to escape from $v$.

When the diameter of $G$ is 3 but $G$ is bipartite, extra movements are needed. Say $V(G)=L \cup R$ is the bi-partition. Without loss of generality, say $v \in L$. All its neighbors $v_{1}, v_{2}, \ldots, v_{l}$ are in $R$. There are three cases:

Case 1: At least $l$ cop positions say $u_{1}, u_{2}, \ldots, u_{l}$ are in $R$. For each $i$, there is a path $P_{i}$ of length at most 2 connecting $u_{i}$ to $v_{i}$. For $1 \leq i \leq l$, move $i$-th cop at $u_{i}$ along the path $P_{i}$ one step toward $v_{i}$. Each $v_{i}$ is now under the control of $i$-th cops. The robber has no way to escape from $v$.

Case 2: There are $k-1$ cop positions in $R$ and one cop position in $L$ (say $u_{k}$ ). If $l<k$, then the case is covered by case 1 . Assume $l=k$. Since both $v$ and $u_{k}$ are in $L$, there is a path $P$ of length 2 connecting $u_{k}$ and $v$. The middle point of this path $P$ is a neighbor of $v$, say $v_{k}$. The robber can not move into $v_{k}$, which is controlled by $u_{k}$. Since other cops at $u_{1}, u_{2}, \ldots, u_{k-1} \in R$, for each $i \leq k-1$, there is a path $P_{i}$ of length at most 2 connecting $u_{i}$ to $v_{i}$. For $1 \leq i \leq l$, move $i$-th cop at $u_{i}$ along the path $P_{i}$ one step toward $v_{i}$. Each $v_{i}$ is now under the control of $i$-th cops. The robber has no way to escape from $v$.

Case 3: This is the remaining case that cops can be anywhere. Move all $k$ cops to some vertices $u_{1}^{\prime}, u_{2}^{\prime}, \ldots, u_{k}^{\prime}$ in $R$. If the robber is still at $v$, then it will be captured by case 1 . So the robber moves into a neighbor of $v$, say $v_{k} \in R$. Send one cop to chase the robber and other cops stay. The robber has to move into a vertex in $L$. From now on, we maintain the property "at least $k-1$ cops are on the different part of the robber". If the robber never returns to $v$, then will be captured in $H \backslash v$ by induction hypothesis. In both case 1 and case 2 , we can capture the robber while keep $k-1$ cops to stay on the other side of the robber. So eventually, the robber moves back to $v$ while there are $k-1$ cops in the other side $R$. By case 1 and 2, the robber will be cornered at $v$ in the next move.

After at most two more steps, the robber is captured at $v$. The induction step is finished.

In the following proof, we will use the concept " $k$-core". The $k$-core of a graph $G$ is the maximum subgraph of $G$ with minimum degree at least $k$. The $k$-core is unique and can be obtained by sequentially deleting all vertices with degrees less than $k$.

Proof of Theorem 1: Fix a small constant $\epsilon>0$ and an integer $k$. Let $p=\frac{\epsilon}{k+1}$ be a probability. Let $G_{0}=G$ and $H_{0}$ be the $k$-core of $G_{0}$. Now, for each vertex $v$ in $H_{0}$, we put one cop with probability $p$ independently. Delete 
the vertices occupied by these cops and their neighbors from $H_{0}$. Denote the remaining graph by $G_{1}$. Now let $H_{1}$ be the $k$-core of $G_{1}$.

For each $i$, let $H_{i}$ be the $k$-core of $G_{i}$. For each vertex $v$ of $H_{i}$, with probability $p$ independently, put one cop at vertex $v$. Now delete the vertices occupied by these cops and their neighbors from $H_{i}$. Denote the remaining graph by $G_{i+1}$. Repeat this process until $G_{i}$ or $H_{i}$ is the empty graph.

Let $H$ be the induced subgraph of $G$ on $\cup_{i \geq 0}\left(G_{i} \backslash H_{i}\right)$. Note that all vertices in $V(G) \backslash V(H)$ are controlled by cops. (Each vertex is either occupied by a cop or one of its neighbors is occupied by a cop.) The robber is forced to move along the edges of $H$. From the construction, it is clear $H$ is a $k$-1-degenerated subgraph. Apply Lemma 3, extra $k-1$ cops can capture the robber in finite steps.

It remains to estimate the total number of the cops needed. For $i \geq 0$, the expected number of cops needed at $i$-th step is by $p \mathbf{E}\left(\left|V\left(H_{i}\right)\right|\right)$. The probability that a vertex $v$ of $H_{i}$ is survived at $G_{i+1}$ is at most

$$
(1-p)^{1+d_{H_{i}}(v)} \leq(1-p)^{k+1}<e^{-p(k+1)} .
$$

Thus the expected number of vertice in $H_{i+1}$ is

$$
\begin{aligned}
\mathbf{E}\left(\left|V\left(H_{i+1}\right)\right|\right) & \leq \mathbf{E}\left(\left|V\left(G_{i+1}\right)\right|\right) \\
& \leq e^{-p(k+1)} \mathbf{E}\left(\left|V\left(H_{i}\right)\right|\right) \\
& \leq \cdots \\
& \leq e^{-(i+1) p(k+1)} \mathbf{E}\left(\left|V\left(H_{0}\right)\right|\right) \\
& \leq e^{-(i+1) p(k+1)} n .
\end{aligned}
$$

The expected number of cops needed in total is at most

$$
k-1+\sum_{i \geq 0} e^{-i p(k+1)} n p .
$$

There is a random instance so that the total cops needed is at most the expected number. We have

$$
\begin{aligned}
c(G) & \leq k-1+\sum_{i \geq 0} e^{-i p(k+1)} n p \\
& =k-1+\frac{n p}{1-e^{-p(k+1)}} \\
& =k-1+\frac{\epsilon}{1-e^{-\epsilon}} \frac{n}{k+1} .
\end{aligned}
$$


Let $f(x)=\frac{x}{1-e^{-x}}$. Choose $k=\lceil\sqrt{f(\epsilon) n}\rceil-1$ to minimize the value. We have

$$
\begin{aligned}
c(G) & \leq k-1+f(\epsilon) \frac{n}{k+1} \\
& \leq\lceil\sqrt{f(\epsilon) n}\rceil-2+f(\epsilon) \frac{n}{\lceil\sqrt{f(\epsilon) n}\rceil} \\
& \leq \sqrt{f(\epsilon) n}-1+f(\epsilon) \frac{n}{\sqrt{f(\epsilon) n}} \\
& =2 \sqrt{f(\epsilon) n}-1 .
\end{aligned}
$$

It holds for any $\epsilon>0$. Let $\epsilon \rightarrow 0$. We have $f(\epsilon) \rightarrow 1$. Thus we get

$$
c(G) \leq 2 \sqrt{n}-1 .
$$

\section{General graphs}

We will prove the following theorem first. Here we make no effort to get the best constant or to remove log-factors.

Theorem 3 Suppose $G$ is a connected graph on $n$ vertices. For any positive integer $k<n$, let $M_{k}=\min _{v \in V(G)}\left|N_{2^{k-1}}(v)\right|$. Then we have

$$
c(G) \leq 8 k\left(\frac{\ln n}{M_{k}}\right)^{\frac{1}{k}} n .
$$

In particular, if the diameter of $G$ is at most $2^{k-1}$, we have $M_{k}=n$. Thus,

$$
c(G) \leq 8 k n^{\frac{k-1}{k}} \ln ^{\frac{1}{k}}(n) .
$$

Remark: Note that, $M_{1}=\delta+1$, where $\delta$ is the minimum degree of $G$. So $M_{k}$ can be viewed as a generalization of $\delta$.

Proof: When $k=1$, we have for any vertex $v$,

$$
|N(v)| \geq M_{1}
$$

Choose $p=\frac{\ln n}{M_{1}}$. For each vertex $v$, with probability $p$, mark $v$ as a cop position independently. Send cops to these cop positions. A vertex $v$ is not controlled by these cops if $N(v)$ contains no cop position. Send additional cops to cover vertices which are not controlled by cops at cop positions. The expected number 
of total cops needed is $n p+\sum_{v}(1-p)^{|N(v)|}$. We have

$$
\begin{aligned}
c(G) & \leq n p+\sum_{v}(1-p)^{|N(v)|} \\
& \leq n p+\sum_{v}(1-p)^{M_{1}} \\
& \leq n p+n e^{-p M_{1}} \\
& \leq n \frac{\ln n}{M_{1}}+1 \\
& <8 n \frac{\ln n}{M_{1}} .
\end{aligned}
$$

So there is a random instance such that $c(G) \leq 8 n \frac{\ln n}{M_{1}}$. The statement holds in this case.

From now on we assume $k \geq 2$. If $8 k\left(\frac{\ln n}{M_{k}}\right)^{\frac{1}{k}}>1$, the statement is trivial since $c(G) \leq n$. We can assume $8 k\left(\frac{\ln n}{M_{k}}\right)^{\frac{1}{k}} \leq 1$. Let $p=4\left(\frac{\ln n}{M}\right)^{\frac{1}{k}} \leq \frac{1}{2 k}$ be a probability. The player $\mathcal{C}$ define a set of cop positions $R$ randomly. For each vertex $v$, with probability $p$, independently mark $v$ as a cop position. Move these cops into cop positions. After finite steps we may assume each cop position is covered by $k$ cops. We form $k$ groups of cops so that each cop position has one cop in each group. (The remaining cops is not used in the later game.)

Suppose the robber is at the vertex $u$. Let $T_{1}(u)=\Gamma^{G}(u)$. We define a bad event $F_{0}(u)$ to be that $d_{u} \geq \frac{2}{p} \ln n$ and there is no cop in $N^{G}(u)=T_{1}(u) \cap\{u\}$. Then,

$$
\operatorname{Pr}\left(F_{0}(u)\right)=(1-p)^{1+d_{u}} \leq e^{-p d_{u}} \leq \frac{1}{n^{2}} .
$$

Except for the bad event $F_{0}(u)$, we have

$$
\left|T_{1}(u)\right|=d_{u}<\frac{2}{p} \ln n
$$

The second group of cops try to control vertices in $T_{1}(u)$ as much as possible after one step. In order to control $T_{1}(u)$ after 1 step, of course, only the cops in $N_{2}\left(T_{1}(u)\right)$ are useful in this purpose. Consider an axillary bipartite graph $H_{1}$ with the partition $T_{1}(u) \cup\left(N_{2}\left(T_{1}(u)\right) \cap R\right)$. For any vertex $v \in N_{1}(u)$ and $y \in\left(N_{2}\left(T_{1}(u)\right) \cap R\right), v y$ is an edge in $H_{1}$ if and only if $d^{G}(u, v) \leq 2$. Apply Lemma 2 to $H_{1}$. There is a matching from $N_{2}\left(T_{1}(u)\right) \cap R$ to $T_{1}(u) \backslash S_{1}(u)$. Here $S_{1}(u) \subset \Gamma(v)$ satisfying

$$
\left|N_{2}\left(S_{1}(u)\right) \cap R\right|<\left|S_{1}(u)\right| \text {. }
$$

Each edge in the matching of $H$ is actually a path of length at most 2 connecting a vertex in $N_{2}\left(T_{1}(u)\right) \cap R$ and a vertex in $T_{1}(u)$. Move the second group of cops on some vertices $N_{2}\left(T_{1}(u)\right) \cap R$ along these paths by one step. They can prevent the robber from entering the vertices in $T_{1}(u) \backslash S_{1}(u)$. 
For any $S \subset T_{1}(u)$, we say $S$ is bad if $\left|N_{2}(S)\right|>\frac{8}{p^{2}} \ln n$ and $\left|N_{2}(S) \cap R\right|<|S|$. Let $X=N_{2}(S) \cap R$, which follows the binomial distribution $B\left(\left|N_{2}(S)\right|, p\right)$. Apply the following Chernoff inequality to $X$ with $a=\mathbf{E}(X)-|S|$. We have

$$
\begin{aligned}
\operatorname{Pr}(X<|S|) & =\operatorname{Pr}(X<\mathbf{E}(X)-a) \\
& \leq e^{-\frac{a^{2}}{2 \mathbf{E}(X)}} \\
& =e^{-\frac{(\mathbf{E}(X)-|S|)^{2}}{2 \mathbf{E}(X)}} .
\end{aligned}
$$

Note that

$$
|S| \leq\left|T_{1}(u)\right|<\frac{2}{p} \ln n<\frac{1}{4} \mathbf{E}(X) .
$$

and then

$$
\mathbf{E}(X)-|S|>\frac{3}{4} \mathbf{E}(X) .
$$

So we have

$$
\begin{aligned}
\operatorname{Pr}(X<|S|) & \leq e^{-\frac{(\mathbf{E}(X)-|S|)^{2}}{2 \mathbf{E}(X)}} \\
& <e^{-\frac{\left(\frac{3}{4} \mathbf{E}(X)\right)^{2}}{2 \mathbf{E}(X)}} \\
& <e^{-\frac{9}{32} \mathbf{E}(X)} .
\end{aligned}
$$

We define a bad event $F_{1}(u)$ to be the event there exists a bad subset $S=$ $S_{1}(u)$ of $T_{1}(u)$. Then

$$
\begin{aligned}
\operatorname{Pr}\left(F_{1}(u) \backslash F_{0}(u)\right) & \leq 2^{\left|T_{1}(u)\right|} e^{-\frac{9 \mathbf{E}(X)}{32}} \\
& =e^{-\frac{9 \mathbf{E}(X)}{32}+\ln 2\left|T_{1}(u)\right|} \\
& <e^{-\left(\frac{9}{4}-2 \ln 2\right) \frac{\ln n}{p}} \\
& <\frac{1}{n^{2}} .
\end{aligned}
$$

At the last step, we apply $p \leq \frac{1}{2 k} \leq \frac{1}{4}$.

Except for the bad events $F_{0}(u) \cup F_{1}(u)$ (with $\left.\operatorname{Pr}\left(F_{0}(u) \cup F_{1}(u)\right) \leq \frac{2}{n^{2}}\right)$, we have

$$
\left|N_{2}\left(S_{1}(u)\right)\right| \leq \frac{8}{p^{2}} \ln n
$$

The robber has to go through some vertex from $S_{1}(u)$ in order to escape from $u$. Let $G_{1}=G \backslash\left(T_{1}(u) \backslash S_{1}(u)\right)$. Let $T_{2}(u)=\Gamma_{3}^{G_{1}}(u)$ be the set of vertices which can be used by the third group of cops to control the neighbors of $S_{1}(u)$ after one step.

For $1 \leq i \leq k-1$, we can recursively define graphs $G_{i}$, sets $T_{i}(u), S_{i}(u)$ and bad events $F_{i}(u)$ as follows.

$$
T_{i}(u)=\Gamma_{2^{i}-1}^{G_{i-1}}(u) .
$$

We define a bad event $F_{i}(u)$ to be the event that there is a subset $S \in T_{i}(u)$ such that $N_{2^{i+1}}(S)>2 \frac{4^{i+1}}{p^{i+2}} \ln n$ and $\left|N_{2^{i+1}}(S) \cap R\right|<|S|$. 
The $i+1$-th group of cops can control $T_{i}(u)$ in $2^{i}-1$ steps except for a subset $S_{i}(u) \subset T_{i}(u)$ satisfying

$$
\left|N_{2^{i}}\left(S_{i}(u)\right) \cap R\right|<\left|S_{i}(u)\right| .
$$

Now we set $G_{i}=G_{i-1} \backslash\left(T_{i}(u) \backslash S_{i}(u)\right)$.

Claim For any $i=1, \ldots, k-1$, except for the bad event $\cup_{j=0}^{i} F_{i}(u)$ (with $\left.\operatorname{Pr}\left(\cup_{j=0}^{i} F_{i}(u)\right)<\frac{i+1}{n^{2}}\right)$, we have

$$
\begin{aligned}
\left|T_{i}(u)\right| & \leq 2 \frac{4^{i-1}}{p^{i}} \ln n ; \\
\left|N_{2^{i}}\left(S_{i}(u)\right)\right| & \leq 2 \frac{4^{i}}{p^{i+1}} \ln n .
\end{aligned}
$$

We will use the induction on $i$ to prove the claim. It holds for $i=1$. Now we assume that it holds for $i$. For $i+1$, we have

$$
T_{i+1}=\Gamma_{2^{i+1}-1}^{G_{i}}(u) \subset N_{2^{i}}\left(S_{i}(u)\right) .
$$

Thus,

$$
\left|T_{i+1}\right| \leq\left|N_{2^{i}}\left(S_{i}(u)\right)\right| \leq 2 \frac{4^{i}}{p^{i+1}} \ln n .
$$

For any $S \subset T_{i+1}$, we say $S$ is bad if $\left|N_{2^{i+1}}(S)\right|>2 \frac{4^{i+1}}{p^{i+2}} \ln n$ and $\mid N_{2^{i+1}}(S) \cap$ $R|<| S \mid$. Let $X=N_{2^{i+1}}(S) \cap R$, which follows the binomial distribution $B\left(\left|N_{2^{i+1}}(S)\right|, p\right)$. Note that

$$
\mathbf{E}(X)=\left|N_{2^{i+1}}(S)\right| p>2 \frac{4^{i+1}}{p^{i+1}} \ln n>4|S| .
$$

Apply Chernoff's inequality to $X$ with $a=\mathbf{E}(x)-|S|>\frac{3}{4} \mathbf{E}(x)$.

$$
\begin{aligned}
\operatorname{Pr}(X<|S|) & =\operatorname{Pr}(X<\mathbf{E}(X)-a) \\
& \leq e^{-\frac{a^{2}}{2 \mathbf{E}(X)}} \\
& \leq e^{-\frac{9}{32} \mathbf{E}(X)}
\end{aligned}
$$

Suppose $\left|T_{i+1}\right| \leq 2 \frac{4^{i}}{p^{i+1}} \ln n$, the probability that there exists a bad subset $S$ is at most

$$
2^{\left|T_{i+1}\right|} e^{-\frac{9}{32} \mathbf{E}(X)}<e^{-\left(\frac{9}{4}-\ln 2\right) 2 \frac{4^{i}}{p^{i+1}} \ln n}<\frac{1}{n^{2}} .
$$

Thus, we have

$$
\begin{aligned}
\operatorname{Pr}\left(\cup_{j=0}^{i+1} F_{j}(u)\right) & =\operatorname{Pr}\left(\cup_{j=0}^{i} F_{j}(u)\right)+\operatorname{Pr}\left(F_{i+1}(u) \backslash \cup_{j=0}^{i} F_{j}(u)\right) \\
& <\frac{i+1}{n^{2}}+\frac{1}{n^{2}} \\
& =\frac{i+2}{n^{2}} .
\end{aligned}
$$


Except for the bad event $\cup_{j=0}^{i+1} F_{j}(u)$, we have

$$
\left|N_{2^{i+1}}\left(S_{i+1}\right)\right| \leq 2 \frac{4^{i+1}}{p^{i+2}} \ln n .
$$

The inductive step is finished.

The last step $i=k-1$ is special. Since for any $u,\left|N_{2^{k-1}}(u)\right| \geq M_{k}$. From the choice of $p$, we have

$$
M_{k}>\frac{4^{k}}{p^{k}} \ln n>\frac{2}{p}\left|T_{k-1}(u)\right| .
$$

Let $F_{k}(u)$ be a bad event that there exists $v \in T_{k-1}(u)$ with $\left|N_{2^{k-1}}(v) \cap R\right|<$ $\left|T_{k-1}(u)\right|$. The claim reads, except for the bad event $\cup_{i=0}^{k-1} F_{k}(u)$,

$$
\left|N_{2^{k-1}}(v)\right| \leq 2 \frac{4^{k-1}}{p^{k}} \ln n .
$$

Apply the Chernoff's equality to the random variable $X=N_{2^{k-1}}(v) \cap R$, which is a sum of some independent $0-1$ random variables. Note

$$
\mathbf{E}(X)=\left|N_{2^{k-1}}(v)\right| p \geq \frac{4^{k}}{p^{k-1}} \ln n .
$$

Choose $\lambda=2 \frac{4^{k-1}}{p^{k-1}} \ln n$ so that

$$
\mathbf{E}(X)-\lambda>\left|T_{k-1}(u)\right|
$$

We have

$$
\begin{aligned}
\operatorname{Pr}\left(\cup_{j=0}^{k} F_{j}(u)\right) & =\operatorname{Pr}\left(\cup_{j=0}^{k-1} F_{j}(u)\right)+\operatorname{Pr}\left(F_{k}(u) \backslash \cup_{j=0}^{k-1} F_{j}(u)\right) \\
& <\frac{k}{n^{2}}+\operatorname{Pr}\left(X<\left|T_{k-1}(u)\right|\right) \\
& <\frac{k}{n^{2}}+\operatorname{Pr}(X<\mathbf{E}(X)-\lambda) \\
& <\frac{k}{n^{2}}+e^{-\frac{\lambda^{2}}{2 \mathbf{E}(X)}} \\
& <\frac{k}{n^{2}}+e^{-\frac{4^{k-1}}{2 p^{k-1}} \ln n} \\
& =\frac{k+1}{n^{2}}
\end{aligned}
$$

At the last step, we applied

$$
\frac{4^{k-1}}{2 p^{k-1}} \geq \frac{2}{p} \geq 2
$$

since $p \leq \frac{1}{4 k}$ and $k \geq 2$. 
Except for the bad event $\cup_{i=0}^{k} F_{i}(u)$, for all $v \in T_{k-1}(u)$, we have

$$
\left|N_{2^{k-1}}(v) \cap R\right|>\left|T_{k-1}(u)\right| \text {. }
$$

We suppose $T_{k-1}(u)=\left\{v_{1}, v_{2}, \ldots, v_{t}\right\}$. For each $i$, we can find a cop $u_{i}$ from $N_{2^{k-1}}\left(v_{i}\right) \cap R \backslash\left\{u_{1}, u_{2}, \ldots, u_{i-1}\right\}$ to move toward $v_{i}$. Therefore, for any vertex of $T_{k-1}$, there is a cop from $k$-th group can control it in less than $2^{k-1}$ steps. No exception set exists. We have

$$
S_{k-1}=\emptyset
$$

Let $U=\{u\} \cup \cup_{i=1}^{k-1}\left(T_{i}(u) \backslash S_{i}(u)\right)$. Every vertex $v$ in $U$ can be controlled by a cop before the robber reaches $v$. Let $K=\cup_{i=1}^{k-2} N_{2^{i}}\left(S_{i}(u)\right)$. Then $K$ forms a cut of a graph $G$ and the robber is in restricted to move inside it. Clearly,

$$
\cup_{i=1}^{k-2} N_{2^{i}}^{G_{i-1}}\left(S_{i}(u)\right) \subset \cup_{i=1}^{k-2} N_{2^{i}}\left(S_{i}(u)\right) .
$$

For this fixed vertex $u$, except for the bad event $\cup_{i=0}^{k-1} F_{i}(u)$, we have

$$
\begin{aligned}
|K| & \leq \sum_{i=1}^{k-2}\left|N_{2^{i}}\left(S_{i}(u)\right)\right| \\
& \leq \sum_{i=1}^{k-2} 2 \frac{4^{i}}{p^{i+1}} \ln n \\
& \leq k \frac{4^{k-1}}{p^{k-1}} \ln n \\
& =k p \frac{4^{k-1}}{p^{k}} \ln n \\
& <k p \frac{4^{k}}{p^{k}} \ln n \\
& =k n p .
\end{aligned}
$$

The expected number of cops we used is knp. With Chernoff inequality, it is clear the number of cops is at most $2 k n p$ with probability at least $1-\frac{1}{n}$.

Next, we show the strategy for the player cop $\mathcal{R}$ to catch the robber using $2 k p$ cops. Clearly, after less than $2^{k-1}$ steps, all vertices in $U$ is under control of cops using at most $k n p$ cops. Let these cops stay at their current position and let other cops to sweep $M$. Here, $|M|<k n p$ guarantee the player cop $\mathcal{R}$ is able to do that. Then, after finite steps, the robber will be caught.

With any choice of $u$, the total probability of bad events is

$$
\sum_{u} \operatorname{Pr}\left(\cup_{i=0}^{k-1} F_{i}(u)\right)+\frac{1}{n} \leq \frac{k+1}{n}<1 .
$$

With positive probability, there is a set of cop position $R$ so that the cops can capture the robber in a finite steps. Thus we have

$$
c(G) \leq 2 k n p=8\left(\frac{\ln n}{M}\right)^{\frac{1}{k}} \leq \frac{1}{2 k} .
$$


If the diameter of $G$ is at most $2^{k-1}$, then we have $M_{k}=n$. The last statement in the theorem follows straightforward.

Proof of Theorem 2 : We use Theorem 3 to prove it. Let $f(x)=8 k\left(\frac{\ln x}{x}\right)^{\frac{1}{k}} x$. Here, take $k=\sqrt{\log _{2} x}$. We prove $c(G) \leq f(n)$ by induction on $n$, the number of vertices of $G$.

When $n=2$. Then $k=1$ and $M_{1}=2$. By Theorem 3 , we have $c(G) \leq 8 \ln 2$. Trivially, it is true.

Suppose it is true for all $G$ such that $|G|<n$.

When $|G|=n$. If the diameter of $G$ is at most $2^{k-1}$. Then by Theorem 3 , we have $c(G) \leq f(n)=8 n \sqrt{\log _{2} n}\left(\frac{\ln n}{n}\right)^{\frac{1}{\sqrt{\log _{2} n}}}$.

If the diameter of $G$ is greater that $2^{k-1}$. Then we have a shortest path, say $P$, with length $2^{k-1}$. At the beginning of the game, we let one cop move along $P$. By Lemma 1, after finite steps, the robber is forbidden to enter this path. We delete $P$ and continue the game on the resulted graph $G-P$. By the induction hypothesis, we have

$$
c(G) \leq c(G-P)+1
$$

Applying the induction hypothesis, we have

$$
c(G) \leq c(G-P)+1 \leq f\left(n-2^{k-1}\right)+1 \leq f(n) .
$$

Here, the last inequality follows from

$$
\begin{aligned}
& f(x)-f\left(x-2^{\sqrt{\log _{2} x}-1}\right)=f^{\prime}(\theta) 2^{\sqrt{\log _{2} x}-1}\left(\text { where } x-2^{\sqrt{\log _{2} x}-1}<\theta<x\right) \\
& >f^{\prime}(x) 2^{\sqrt{\log _{2} x}-1}>1 \text {. }
\end{aligned}
$$

when $x \geq 3$. Clearly, $f(n)=O\left(\frac{n}{2^{(1-o(1)) \sqrt{\log _{2} n}}}\right)$ when $n$ large enough. We completed the proof of Theorem 3 .

Acknowledgement: The first author thanks Anthony Bonato and PawełPrałat for useful discussion on the cop number of random graphs and for introducing the Meyniel's Conjecture.

\section{References}

[1] M. Aigner and M. Fromme, A game of cops and robbers, Discrete Appl Math 8 (1984), 1-12.

[2] B. Alspach, Searching and sweeping graphs: A brief survey, Le Matematiche 59 (2004), 5-37.

[3] T. Andreae, On a pursuit game played on graphs for which a minor is excluded, J. Combin Theory B 41(1) (1986), 37-47. 
[4] A. Berarducci and B. Intrigila, On the cop number of a graph, Adv. Appl. Math. 14(4) (1993), 389-403.

[5] P. Frankl, Cops and robbers in graphs with large girth and cayley graphs, Discrete Appl. Math. 17(3) (1987), 301-305.

[6] B. Bollobás, G. Kun and I. Leader, Cops and robbers in a random graph, preprint.

[7] Ehsan Chiniforooshan, A better bound for the cop number of general graphs, J. of Graph Theory 58(1) (2008) 45-48.

[8] P. Frankl, Cops and robbers in graphs with large girth and cayley graphs, Discrete Appl Math 17(3) (1987), 301-305.

[9] R. J. Nowakowki and P. Winkler, Vertex to vertex pursuit in a graph, Discrete Math. 43 (1983), 235-239.

[10] A. Quilliot, A probèmes de jeux de point fixe, de connectivit'e et de représentation sur des graphes des ensembles ordonnés et des hypergraphes, Thèse d'Etat, Université de Paris VI, 1983.

[11] A. Quilliot, A short note about pursuit games played on a graph with a given genus, J. Combin. Theory B 38 (1985), 89-92.

[12] T. Łuczak and P. Prałat, Chasing robbers on random graphs: zigzag theorem, preprint. 